\title{
Correction to: Recent Advances and Future Directions: Clinical Applications of Intraoperative BOLD-MRI CVR
}

\author{
Giovanni Muscas, Christiaan Hendrik Bas van Niftrik, Martina Sebök, \\ Alessandro Della Puppa, Luca Regli, and Jorn Fierstra
}

Correction to:

Chapter 11 in: Jean Chen and Jorn Fierstra (eds.), Cevebrovascular Reactivity: Methodological Advances and Clinical Applications, Neuromethods, vol. 175, https://doi.org/10.1007/978-1-0716-1763-2_11

The authors name Alessandro Della Puppa is wrongly listed as "Puppa A.D” and this has been updated. 